\title{
A Review on Deep Convolutional Neural Network and its Applications
}

\author{
Sugandhi Maheshwaram ${ }^{1}$ \\ Senior Full Stack Developer, National Association of Insurance Commissioners (NAIC), Kansas City, Kansas, \\ $\mathrm{USA}^{1}$
}

\begin{abstract}
Conventional architectures made use of for dealing computer system vision issues are greatly in control on customer attributes. Yet the brand-new deep discovering strategies have actually offered a replacement for instantly finding out the issue- associated attributes. Therefore the understanding of what kind of deep networks appropriate for an offered issue collection is a tough job. Convolutional Semantic Network (CNN) was to start with introductory in Computer system Vision for picture acknowledgment by Le- Cun et al. in 1989. Ever since it has actually been commonly utilized in photo acknowledgment and also category jobs The current outstanding success of Krizhevsky et al. in ILSVRC 2012 competitors show the considerable development of modern deep CNN on picture category job. Influenced by his job, several current study jobs have actually been concentrat- ing on comprehending CNN as well as prolonging its application to even more traditional computer system vision jobs. Their successes and also lessons have actually advertised the growth of both CNN and also vision science research. This short article makes a study of current development in CNN because in 2012. We will certainly present the general architecture of a contemporary CNN as well as make understandings right into numerous common CNN versions which have actually been researched thoroughly. We will certainly additionally evaluate the initiatives to comprehend $\mathrm{CNN}$ as well as evaluation essential applications of CNN's in computer system vision jobs..
\end{abstract}

Index Terms : Deep Learning, Convolutional Deep Belief Network, Convolutional Neural Networks, Natural Language, Computer Vision

\section{INTRODUCTION}

The deep convolutional semantic network was educated on ImageNet dataset with 1.2 million classified pictures with information enhancement, as well as structured with customized layers such as ReLU as well as Failure. They additionally utilized a lot more effective equipment to do float calculations. These concepts, as well as methods, have actually laid the foundation for contemporary convolutional semantic networks. Ever since increasingly more study jobs have actually revealed the significant development of CNN's over modern state-of-art approaches on big datasets. Today, convolutional semantic networks have actually been effectively gotten item detection, photo category, photo division, movement discovery as well as inter- corrective jobs of computer system vision and also all-natural language handling. Meantime, open resource deep understanding devices such as Caffe have actually been emerging and also accumulating features from one of the most current study jobs. This gives one more propulsion of the create- meant of deep knowledge in computer system vision

An Artificial Semantic Network [4] is an information handling archetype that is affected by the working of organic sensory systems, such as the mind procedure info. The vital part of this standard is the unique framework of the details taking care of structure. It is constructed out of a considerable variety of really interconnected taking care of parts (nerve cells) functioning as one to deal with specific concerns. Understanding the treatment of ANN resembles human beings, picking up from a variety of instances. An ANN is created for a certain application, for instance, pattern acknowledgment or information category, via a knowing treatment. Knowing in this structures consists of adjustments according to the short organizations that exist in between the nerve cells.

A Convolutional Semantic Network (CNN) contain greater than one convolutional layer with a subsampling layer and also afterward took after by at the very least one entirely connected layers as in a common multilayer system. All type of 2D information such as picture or speech signal can be refined with the architecture of the convolutional semantic network. This is acquired by weight, neighborhood link, as well as pooling. Neural network systems, with their incredible ability to obtain value from knotted or loosened details, can be made use of to focus layouts as well as identify patterns that are also uncertain to ever before be seen by either individuals or various other computational methods. 


\author{
Vol. 8, Issue 2, February 2019
}

The principle of a semantic network has actually currently existed because the 1950s when Frank Rosenblatt produced the perception. Also, the convolutional semantic network itself is not a brand-new disadvantage whatsoever. The convolutional semantic network was to start with presented in [3] to identify transcribed POSTAL CODE in 1989, as well as later on included acknowledgment as well as category of different things such as hand-written figures (MNIST) [4], home numbers, web traffic indications as well as much more lately 1000-category ImageNet dataset.

\title{
II. LITERATURE SURVEY
}

CireAn, Dan, Ueli Meier, Jonathan Masci, as well as Jürgen Schmidhuber discusses regarding the application of a computerized system that can be qualified to identify the German web traffic indication. The efficient use of parameterized GPU for the application of DCNN [9] boosted the effectiveness of approximately 99.46\%. Rather than utilizing one DCNN for the whole procedure, they incorporated numerous DNN that educated under various problems right into numerous columns. And also thus it accomplishes the highest possible precision also when it comes to lighting as well as comparison adjustments. This technique can be embraced for identifying any kind of various other web traffic indications. The foundation of their job is Multi-column deep neural network( MCD) [10] It changes the convolutions with max merging [11] layers in the conventional architecture. According to the architecture, preprocessed the input picture by $n$ various preprocessors and also an arbitrary variety of sections prepared on every area input. The averaging specific insight of each DNN provides the last forecast. Each DNN component has a team of convolutional as well as max-pooling layers. Convolutional layer carries out the 2D convolution, as well as the outcomes, are traveled through the activation feature. Making use of max merging layer instead of subsampling layer sets apart DNN from CNN. The dimension of limit merging, as well as convolutional bits, are picked in a manner that the result maps to a 1D attribute vector. Dataset is preprocessed well prior to training. The arbitrary minimal esteem for utilizing translation, turning, scaling is picked such a manner in which $\pm 10 \%$ of the initial dimension of the picture and also \pm 5 levels for turning. The various DNN column is designated with various weight. One input can be defined differently. Lastly, the outcome acquiring from each DNN is balanced. This will certainly decrease the ordinary mistake. They made use of a system with core i7-- 950 (3.33 GHz), 24 GB DDR3, as well as 4 graphics cards of kind GTX 580 and also 9 DNN layer framework for executing this job. Which can be prepared by utilizing easy slope descend. Each layer includes 25 webs for 5 various information. The outcome accomplishes the acknowledgment price of $99.46 \%$. Comparing with specific DNN it is an extreme success. Integrating the private DNN boosts the precision along with rises the effectiveness of a different kind of sound.

Imagenet is a photo data source made up by the globe internet tiers in which each node is represented by thousands of photos. Krizhevsky, Alex, Ilya Sutskever, as well as Geoffrey E. Hinton [4] describes the range of DCNN in the area of huge information. Below CNN is utilized to categorize the 1.2 numerous the top-notch photo right into greater than 1000 groups [12] It mentions they might attain the most affordable mistake price amongst existing approach. The efficient monitoring of CPU system minimizes the moment needed for performing. To boost the efficiency of the classifier in a huge dataset, find out the a lot more effective version as well as discover the most effective approach to lower the overfitting [13] convolutional semantic networks are the most effective selection as a result of its effectiveness in taking care of huge datasets as well as top quality to decrease overfitting. It is a really boosted GPU use of 2D convolution [14]

Zou, Yuexian, Bouquet Li, Yi Wang, Jiasheng Yu, Yi Li, and also W. J. Deng is based upon the cordless pill endoscopy photos by utilizing the capacity of effective knowing ability o DCNN it is made use of to divide the high semantic functions from WCE pictures. It has actually accomplished the $95 \%$ of precision by educating the system with countless photos with different criteria. A cordless mini enveloped camera is made use of to catch photos inside the tummy. The electronic camera takes 50000 to 60000 pictures per 8 hrs of a research study. It is noninvasive, pain-free and also not reusable. By utilizing these photos the DCNN system categorizes the digestion body organs right into the belly, little intestinal tract as well as the colon. There exist a number of techniques to categorize these organs-by making use of DCT as well as PCA making use of Getting Operating Particular (ROC) contour evaluation [6], category utilizing border based upon power feature [7] However this job focuses on the ability of the DCNN for category.

\section{NEURAL NETWORK ARCHITECTURES}

Though it has actually more than 25 years after the initial disadvantage- the evolutional semantic network was suggested, modern-day convolutional semantic networks still share really comparable architectures with the initial one, such as convolutional layers, as well as share comparable training plan such as ahead and also backward proliferation. In this area, we will certainly offer an account of a normal convolutional semantic network as well as present one of the most essential suggestions in the style of a convolutional semantic network. In the remainder of this study, we will certainly make use of the term $\mathrm{CNN}$ and also convolutional semantic network reciprocally. 


\title{
IJARCCE
}

\section{International Journal of Advanced Research in Computer and Communication Engineering}

\author{
Vol. 8, Issue 2, February 2019
}

First of all, we will certainly present 3 most import convolutional semantic network versions that often referred to in this paper: LeNet, ConvNet, and also VGGNet. The LeNet was first of all presented in 1989 and also developed to grow in 1998 to identify hand-written figures. LeNet is frequently viewed as the model of a contemporary CNN. Its framework is received Number 1. The ConvNet was suggested by Krizhevsky et al. in 2012 for ImageNet Large-Scale Visual Acknowledgment Obstacle (ILSVRC) com- request, as well as began the age of deep understanding in computer system vision. Those imaginative concepts brought by ConvNet such as information enhancement as well as corrected direct activation feature have actually been verified vital to educating a fantastic range modern-day CNN. Its framework is received Number 2. The VGGNet is a renovation of ConvNet in 2014, as well as its technique people- in much deeper layers and also smaller sized convolutional filters, has actually been commonly approved to enhance efficiency. It is additionally a standard CNN of producing picture deep functions in several deep knowing associated jobs.

Table 1: LeNet, ConvNet and VGGNet Architectures

\begin{tabular}{|c|c|c|}
\hline \multicolumn{3}{|c|}{ Convolutional Neural Networks Configurations } \\
\hline LeNet & ConvNet & VGGNet \\
\hline \begin{tabular}{|l}
5 weight \\
layers
\end{tabular} & $\begin{array}{l}8 \text { weight } \\
\text { layers }\end{array}$ & $\begin{array}{l}16 \text { weight } \\
\text { layers }\end{array}$ \\
\hline $\begin{array}{l}\text { Input } \\
(28 \times 28 \\
\text { gray image })\end{array}$ & \multicolumn{2}{|c|}{$\begin{array}{l}\text { Input } \\
(224 \times 224 \text { RGB image })\end{array}$} \\
\hline \begin{tabular}{|l|} 
conv \\
$6 @ 5 \times 5$
\end{tabular} & $\begin{array}{l}\text { conv } \\
96 @ 11 \times 11\end{array}$ & $\begin{array}{l}\text { conv 64@3x3 } \\
\text { conv 64@3x3 }\end{array}$ \\
\hline \multicolumn{3}{|l|}{ pooling } \\
\hline \begin{tabular}{|l|} 
conv \\
$16 @ 5 \times 5$
\end{tabular} & $\begin{array}{l}\text { conv } \\
256 @ 5 \times 5\end{array}$ & $\begin{array}{l}\text { conv128@3x3 } \\
\text { conv128@3x3 }\end{array}$ \\
\hline \multicolumn{3}{|l|}{ pooling } \\
\hline \multirow{6}{*}{ NA } & conv384@3x3 & $\begin{array}{l}\text { conv256@3x3 } \\
\text { conv256@3x3 conv 256@3x3 }\end{array}$ \\
\hline & \multicolumn{2}{|l|}{ pooling } \\
\hline & conv 384@3x3 & $\begin{array}{l}\text { conv512@3x3 } \\
\text { conv512@3x3 conv 512@3x3 }\end{array}$ \\
\hline & NA & pooling \\
\hline & conv256@3x3 & $\begin{array}{l}\text { conv 512@3x3 } \\
\text { conv512@3x3 conv 512@3x3 }\end{array}$ \\
\hline & \multicolumn{2}{|l|}{ pooling } \\
\hline fc-120 & \multicolumn{2}{|l|}{ fc-4096 } \\
\hline fc-84 & \multicolumn{2}{|l|}{ fc-4096 } \\
\hline fc-10 & \multicolumn{2}{|l|}{ fc-1000 } \\
\hline$\overline{R B F}$ & \multicolumn{2}{|l|}{ softmax } \\
\hline
\end{tabular}

\section{APPLICATIONS OF CNN}

\section{Computer Vision}

Convolutional semantic networks are used to recognize the pecking order or theoretical framework of a photo. Rather than feeding each picture right into the semantic network as one grid of numbers, the picture is damaged down right into overlapping picture floor tiles that are each fed right into a tiny semantic network.

Convolutional semantic networks are trainable multi-stage architectures [3], [4] with the inputs and also outcomes of each phase including collections of varieties called function maps. If the input is a color picture, each attribute map is a $2 \mathrm{D}$ range having a color network of the input photo, for a video clip or a volumetric photo it would certainly be a 3D range. 


\title{
International Journal of Advanced Research in Computer and Communication Engineering
}

\author{
Vol. 8, Issue 2, February 2019
}

Each attribute removed in any way places on the input is stood for by a function map at the result. Each phase is made up of a filter financial institution layer, a non-linearity layer and also a function merging layer. A common CNN is made up of one, 2 or 3 such 3 - layer phases, complied with by a category component.

\section{1) Face Recognition:}

Face Recognition makes up a collection of associated troubles-.

$>$ Recognizing all the faces aware.

$>$ Concentrating on each face regardless of poor illumination or various position.

$>$ Determining special attributes.

$>$ Contrasting recognized functions to existing data source as well as establishing the individual's name.

Faces stand for a complicated, multi-dimensional, aesthetic stimulation which was earlier provided utilizing a crossbreed semantic network integrating regional picture tasting, a self-organizing map semantic network and also a convolutional semantic network. The outcomes existed utilizing Karhunen-Loe've change instead of the self-organizing map which carried out nearly also (5.3\% mistake versus $3.8 \%)$ as well as a multi-layer perceptron which choked up (40\% mistake versus $3.8 \%$ ).

\section{2) Scene Labelling:}

Each pixel is classified with the group of the things it comes from in scene labeling. Clement Farabet et alia recommended a technique utilizing a multiscale convolutional network that generated document precisions on the Sift Circulation Dataset (33 courses) and also the Barcelona Dataset (170 courses) as well as near-record precision on Stanford History Dataset (8 courses). Their approach created 320 X 240 picture labeling in under a 2nd consisting of attribute removal.

Recurring architecture for convolutional semantic network recommends a consecutive collection of networks sharing the exact same collection of criteria. The network instantly discovers to smooth its very own anticipated tags. As the context dimension enhances with the integrated reappearance, the system determines as well as fixes its very own mistakes. An easy and also scalable discovery formula that enhances mean typical accuracy (mAP) by greater than $30 \%$ about the previous finest outcome on VOC 2012-- accomplishing an mAP of 53.3\% was recommended by scientists at UCB and also ICSI. It was called as R-CNN: Areas with CNN includes as it mixed area propositions with CNN attributes. Completely convolutional networks educated end-to-end, pixels- to-pixels deal with the drawbacks of previous methods of CNN's which were made use of for semantic division in which each pixel was identified with the course of its inscribing item or area. Totally convolutional networks adjusted from modern category networks such as AlexNet, GoogleNet and also VGG web attain advanced division of PASCAL VOC (20\% family member enhancement to $62.2 \%$ mean IU on 2012), NYUDv2, as well as SORT Circulation, while reasoning takes much less than one fifth of a 2 nd for a regular photo. [7] Given that the previous 2 years Deep Convolutional Neural Networks (DCNNs) have actually substantially boosted the efficiency of computer system systems on issues in photo category (Krizhevsky et al., 2013; Sermanet et al., 2013; Simonyan \& Zisserman, 2014; Szegedy et al., 2014; Papandreou et al., 2014). The job of George Papandreou, Liang-Chieh Chen et al. reveals that reactions at the last layer of DCNNs are not completely local for precise things division. A completely attached Conditional Radom Area (CRF) is utilized to conquer this trouble as well as provides a $71.6 \%$ IOU precision in the examination established for a brand-new state-of-art at the PASCAL VOC- 2012 semantic picture division job.

\section{3) Image Classification:}

Compared to various other approaches $\mathrm{CNN}$ 's attain much better category precision on huge range datasets as a result of their capacity of a joint attribute as well as classifier discovering. [9]create the AlexNet as well as attain the most effective efficiency in ILSVRC 2012. Adhering to the success of the AlexNet, numerous jobs made substantial enhancements in category precision by decreasing filter dimension or broadening the network deepness.

A quick, totally parameterizable GPU application of CNN released benchmark outcomes for things category (NORB, CIFAR10) with mistake prices of $2.53 \%, 19.51 \%$. GPU code for picture category is up to 2 sizes quicker than its CPU equivalent. Multi-column deep semantic networks(MCDNN) can exceed all previous techniques of picture category as well as show that pre-training is not essential(though often valuable for little datasets) while reducing the mistake price by $30-40 \%$ Non-saturating nerve cells and also effective GPU execution of the convolution procedure led to a winning top-5 examination mistake price of $15.3 \%$, contrasted to $26.2 \%$ attained by the second-best access in the ILSVRC-2012 


\author{
Vol. 8, Issue 2, February 2019
}

competitors for category of 1.2 million high-resolution pictures in the ImageNet LSVRC-2010 competition right into the 1000 various courses.

Ordered Deep Convolutional Neural Networks (HD- CNN) are based upon the instinct that some courses in photo category are extra complicated than various other courses. It improves the standard CNN's which are N-way classifiers as well as adheres to the coarse-to-fine category technique and also layout component. HD-CNN with CIFAR100- NIN foundation is attended reveal a screening precision of $65.33 \%$ which is greater than the precision for various other common deep designs as well as HD-CNN designs on CIFAR100 dataset.

\title{
4) Human Pose Estimation:
}

Human-pose acknowledgment is long-lasting trouble in computer system vision. This is mostly as a result of the high dimensionality of the input information as well as the high irregularity of feasible body positions. Conventional techniques have a tendency to depend upon look hints to anticipate the human present instead of motion-based attributes. Since both the neighborhood proof as well as the international framework are handcrafted, there is a huge range for mistakes. For this reason, this system attempts to find out both the regional functions as well as the worldwide framework utilizing a convolutional semantic network. This system usages motion-based attributes to outshine existing modern strategies to forecast the human present.

\section{CONCLUSION}

$\mathrm{CNN}$ is certainly at its flourish today, as well as it redefines most of the cutting-edge techniques in computer system vision. In this paper, a study based upon an application of a deep convolutional semantic network exists. This job will certainly aid to acquaint the application of neural network carefully. To categorize the photos of big dataset like image-net, a semantic network is well executed. Likewise feasible to club the monitored discovering method like CDBN with deep architecture to improve the knowledge. Making use of the rugged attribute removal ability of the common surprise layer, it is made use of for the personality acknowledgment. Making use of the greater semantic photo attributes, DCNN can be made use of for the real-time category gastrointestinal body organs from cordless sensing unit endoscopy pictures.

\section{REFERENCES}

[1]. A. Berg, J. Deng, and L. Fei-Fei. Large scale visual recognition challenge 2010. www.image- net.org/challenges. 2010.

[2]. L. Breiman. Random forests. Machine learning, 45(1):5-32, 2001.

[3]. D. Cires an, U. Meier, and J. Schmidhuber. Multi-column deep neural networks for image classification. Arxiv preprint arXiv:1202.2745, 2012.

[4]. D.C. Cires san, U. Meier, J. Masci, L.M. Gambardella, and J. Schmidhuber. High-performance neural networks for visual object classification. Arxiv preprint arXiv:1102.0183, 2011.

[5]. J. Deng, W. Dong, R. Socher, L.-J. Li, K. Li, and L. Fei-Fei. ImageNet: A Large-Scale Hierarchical Image Database. In CVPR09, 2009.

[6]. J. Deng, A. Berg, S. Satheesh, H. Su, A. Khosla, and L. Fei-Fei. ILSVRC-2012, $2012 . \quad$ URL

http://www.image-net.org/challenges/LSVRC/2012/.

[7]. L. Fei-Fei, R. Fergus, and P. Perona. Learning generative visual models from few training examples: An incremental bayesian approach tested on 101 object categories. Computer Vision and Image Understand- ing, 106(1):59-70, 2007

[8]. G. Griffin, A. Holub, and P. Perona. Caltech-256 object category dataset. Technical Report 7694, Cali- fornia Institute of Technology, 2007. URL http://authors.library.caltech.edu/7694.

[9]. G.E. Hinton, N. Srivastava, A. Krizhevsky, I. Sutskever, and R.R. Salakhutdinov. Improving neural net- works by preventing co-adaptation of feature detectors. arXiv preprint arXiv:1207.0580, 2012.

[10]. K. Jarrett, K. Kavukcuoglu, M. A. Ranzato, and Y. LeCun. What is the best multi-stage architecture for object recognition? In International Conference on Computer Vision, pages 2146-2153. IEEE, 2009.

[11]. A. Krizhevsky. Learning multiple layers of features from tiny images. Master's thesis, Department of Computer Science, University of Toronto, 2009.

[12]. LeCun, Yann, Koray Kavukcuoglu, and Clément Farabet. "Convolutional networks and applications in vision." ISCAS. 2010.

[13]. Farabet, Clement, et al. "Learning hierarchical features for scene labeling."IEEE transactions on pattern analysis and machine intelligence 35.8 (2013): 1915-1929.

[14]. Pinheiro, Pedro HO, and Ronan Collobert. "Recurrent Convolutional Neural Networks for Scene Labeling." ICML. 2014.

[15]. Girshick, Ross, et al. "Rich feature hierarchies for accurate object detection and semantic segmentation." Proceedings of the IEEE conference on computer vision and pattern recognition. 2014.

[16]. Long, Jonathan, Evan Shelhamer, and Trevor Darrell. "Fully convolutional networks for semantic segmentation." Proceedings of the IEEE Conference on Computer Vision and Pattern Recognition. 2015.

[17]. Chen, Liang-Chieh, et al. "Semantic image segmentation with deep convolutional nets and fully connected crfs."arXiv preprint arXiv:1412.7062(2014)

[18]. Gu, Jiuxiang, et al. "Recent Advances in Convolutional Neural Networks."arXiv preprint arXiv:1512.07108 (2015).

[19]. M. D. Zeiler and R. Fergus, "Visualizing and understanding convolutional networks," in ECCV, 2014.

[20]. Ciresan, Dan C., et al. "Flexible, high performance convolutional neural networks for image classification." IJCAI Proceedings- International Joint Conference on Artificial Intelligence. Vol. 22. No. 1. 2011.

[21]. Yeshwanth Rao Bhandayker, "AN OVERVIEW OF THEINTEGRATION OF ALL DATA MINING AT CLOUD-COMPUTING” in “Airo International Research Journal”, Volume XVI, June 2018 [ISSN : 2320-3714] 


\title{
International Journal of Advanced Research in Computer and Communication Engineering
}

\author{
Vol. 8, Issue 2, February 2019
}

[22]. Siripuri Kiran, 'Decision Tree Analysis Tool with the Design Approach of Probability Density Function towards Uncertain Data Classification', International Journal of Scientific Research in Science and Technology(IJSRST), Print ISSN : 2395-6011, Online ISSN : 2395-602X,Volume 4 Issue 2, pp.829-831, January-February 2018. URL : http://ijsrst.com/IJSRST1841198

[23]. Yeshwanth Rao Bhandayker, "Artificial Intelligence and Big Data for Computer Cyber Security Systems" in "Journal of Advances in Science and Technology", Vol. 12, Issue No. 24, November-2016 [ISSN : 2230-9659]

[24]. Sugandhi Maheshwaram, "A Comprehensive Review on the Implementation of Big Data Solutions" in "International Journal of Information Technology and Management", Vol. XI, Issue No. XVII, November-2016 [ISSN : 2249-4510]

[25]. Ajmera Rajesh, Siripuri Kiran, " Anomaly Detection Using Data Mining Techniques in Social Networking" in "International Journal for Research in Applied Science and Engineering Technology", Volume-6, Issue-II, February 2018, 1268-1272 [ ISSN : 2321-9653], www.ijraset.com

[26]. Sugandhi Maheshwaram, "An Overview of Open Research Issues in Big Data Analytics" in "Journal of Advances in Science and Technology", Vol. 14, Issue No. 2, September-2017 [ISSN : 2230-9659]

[27]. Siripuri Kiran, Ajmera Rajesh, "A Study on Mining Top Utility Itemsets In A Single Phase" in "International Journal for Science and Advance Research in Technology (IJSART)", Volume-4, Issue-2, February-2018, 637-642, [ ISSN(ONLINE): 2395-1052]

[28]. Yeshwanth Rao Bhandayker, "Security Mechanisms for Providing Security to the Network" in "International Journal of Information Technology and Management", Vol. 12, Issue No. 1, February-2017, [ISSN : 2249-4510]

[29]. Sugandhi Maheshwaram, S. Shoban Babu, "An Overview towards the Techniques of Data Mining" in "RESEARCH REVIEW International Journal of Multidisciplinary", Volume-04, Issue-02, February-2019 [ISSN : 2455-3085]

[30]. Yeshwanth Rao Bhandayker, "A Study on the Research Challenges and Trends of Cloud Computing" in "RESEARCH REVIEW International Journal of Multidisciplinary ", Volume-04, Issue-02, February-2019 [ISSN : 2455-3085]

[31]. Sriramoju Ajay Babu, Dr. S. Shoban Babu, "Improving Quality of Content Based Image Retrieval with Graph Based Ranking" in "International Journal of Research and Applications", Volume 1, Issue 1, Jan-Mar 2014 [ ISSN : 2349-0020 ]

[32]. Dr. Shoban Babu Sriramoju, Ramesh Gadde, "A Ranking Model Framework for Multiple Vertical Search Domains" in "International Journal of Research and Applications" Vol 1, Issue 1,Jan-Mar 2014 [ ISSN : 2349-0020 ].

[33]. Mounika Reddy, Avula Deepak, Ekkati Kalyani Dharavath, Kranthi Gande, Shoban Sriramoju, "Risk-Aware Response Answer for Mitigating Painter Routing Attacks" in "International Journal of Information Technology and Management", Volume VI, Issue I, Feb 2014 [ ISSN : 2249-4510] 